\title{
Propriedades físicas e mecânicas de painéis de lâminas paralelas (PLP) produzidos com madeira de Hevea brasiliensis
}

\author{
Douglas Lamounier Faria*, Lucas Passos Ribeiro, Kleber Magalhães Oliveira, José Benedito Guimarães \\ Júnior
}

Universidade Federal de Lavras (UFLA), Lavras, Minas Gerais, Brasil.

\begin{abstract}
RESUMO O objetivo deste trabalho foi avaliar a viabilidade de utilização da espécie Hevea brasiliensis para produção de painéis de lâminas paralelas (PLP). Os painéis foram produzidos com sete lâminas dispostas na mesma direção da grã, coladas com os adesivos resorcinol-formaldeído (RF) e poliuretano vegetal (PV), com três gramaturas (240, 280 e 320 g.m-m), pressão específica de $1 \mathrm{MPa}$ e temperatura de prensagem ambiente. Em relação aos ensaios físicos realizados, foi avaliada a umidade, densidade aparente e absorção de água dos painéis; já para as propriedades mecânicas, foi avaliada a resistência e rigidez à compressão paralela às fibras e a dureza Janka. Os resultados indicaram diferença estatística dos efeitos principais: a gramatura e tipo de adesivo apenas na absorção de água e no ensaio de compressão paralela. Para todas as propriedades avaliadas, ambos adesivos apresentaram comportamento similar. Os painéis ficaram abaixo do limite máximo de $10 \%$ para resistência à delaminação. Concluiu-se que a espécie apresenta viabilidade técnica para a produção de painéis PLP, podendo minimizar os custos de produção por meio da utilização de menor gramatura de ambos adesivos estudados.
\end{abstract}

Palavras-chave: seringueira; chapas de madeira; construção civil.

\section{Physical and mechanical properties of vertically laminated veneer lumber (LVL) produced with Hevea brasiliensis wood}

\begin{abstract}
The aim of this work was to evaluate the feasibility of Hevea brasiliensis wood species for the production of vertically laminated veneer lumber panels (LVL). The panels were produced with seven veneers arranged in the same grain direction, bonded with resorcinol-formaldehyde (RF) and vegetal polyurethane (PV) adhesives, with three grammages (240, 280 and 320 g.m $\mathrm{m}^{-2}$ ), specific pressure of $1 \mathrm{MPa}$ and ambient pressing temperature. Regarding to the physical tests performed, the moisture, apparent density and water absorption of the panels were evaluated; already for the mechanical properties, it was evaluated the resistance and stiffness to the parallel compression to the fibers and the Janka hardness. The results indicated a statistical difference of the main effects: grammage and type of adhesive only for the water absorption and for the parallel compression tests. For all evaluated properties, both adhesives presented similar behavior. Panels were below the maximum limit of $10 \%$ for resistance to delamination. It was concluded that the species presents technical feasibility for the production of LVL panels, being able to minimize production costs through the use of a lower grammage of both adhesives studied.
\end{abstract}

Keywords: rubber wood; wood boards; civil construction.

\section{Introdução}

O desenvolvimento de novas opções produtivas de maior valor agregado mediante a diversificação de produtos madeireiros é vital para aceder a novos mercados. Assim surgiu uma geração de produtos da madeira, que compreende uma grande variedade de produtos florestais que podem ser intitulados de forma genérica em "produtos engenheirados à base de madeira" (Engineered wood products). São produtos à base de madeira que após transformação, em maior ou menor grau, por um processo industrial que reduz a madeira a frações ou componentes mais elementares ou convenientes, para posterior reassociação objetivando maior performance estrutural (LARA PALMA; BALLARIN, 2011). 
O painel de lâminas paralelas (PLP), é um produto de madeira engenheirada desenvolvido na década de 70 nos Estados Unidos da América, utilizado como componente estrutural de edificações, sobretudo em países onde há tradição no uso de madeira em sistemas construtivos (MÜLLER et al., 2015; IWAKIRI et al., 2016). O PLP apresenta a vantagem de ser um compósito uniforme, mantendo as propriedades originais da madeira (BULIGON et al., 2015). No Brasil a sua produção ainda não ocorre em escala industrial, porém sua aplicação está aos poucos sendo inserida na construção civil. Este é um compósito de madeira laminada sobreposta na mesma direção da grã em camadas unidas por resina.

Vários autores têm estudado diferentes espécies florestais de rápido crescimento, visando viabilizar a aplicação desses painéis na indústria da construção civil brasileira. Lahr et al. (2007) estudaram a estimativa da porcentagem de delaminação de painéis PLP produzidos com o híbrido Eucalyptus grandis Hill ex Maiden X Eucalyptus urophylla S.T. Blake, enquanto Iwakiri et al. (2008) avaliaram a produção de painéis estruturais com madeiras de Eucalyptus grandis Hill ex Maiden e Eucalyptus dunnii Maiden. Ambos os autores obtiveram resultados satisfatórios na avaliação das propriedades físicas e mecânicas. Pesquisas têm mostrado o potencial de utilização de madeiras tropicais na produção de painéis PLP, como observado por Berger et al. (2018) estudaram PLP produzidos com a combinação de lâminas das madeiras de Hovenia dulcis (Cajueiro japonês) e Corymbia citriodora (Eucalipto citriodora), os quais obtiveram satisfatórios resultados nos ensaios de flexão estática e cisalhamento da linha de cola. Mendoza et al. (2017) compararam as propriedades de painéis compensados e painéis PLP de Trattinnickia burserifolia (Amescla), verificaram superioridade em relação ao painel compensado, sendo, uma alternativa viável dentro do setor madeireiro, ampliando a quantidade de produtos disponíveis para o mercado consumidor.
No Brasil ainda são escassas as pesquisas sobre painéis estruturais PLP produzidos com madeiras tropicais. Logo, há a necessidade de estudo sobre espécies madeireiras visando à produção e consolidação desse tipo de painel no mercado brasileiro, como uma alternativa para as estruturas de concreto e aço, que são materiais não renováveis. Como exemplo está a espécie tropical Hevea brasiliensis, que após o ciclo produtivo do látex, as árvores são cortadas e a madeira é tradicionalmente utilizada para carvão e lenha. Com isso, esse trabalho teve como objetivo avaliar o potencial de utilização da espécie Hevea brasiliensis para fabricação de PLP, visando agregar valor à madeira de Hevea brasiliensis.

\section{Material e Métodos}

\section{Obtenção e preparo da matéria-prima}

Para realização deste estudo, foram utilizadas seis árvores do clone de Hevea brasiliensis RRIM 600 com 12 anos de idade, e DAP (diâmetro do fuste à altura de 1,30 $\mathrm{m}$ do solo) médio de $28 \mathrm{~cm}$ em um seringal na Fazenda Carandaí, localizada no município de Nepomuceno, na região sul do estado de Minas Gerais, Brasil, cujas coordenadas geográficas da fazenda são $21^{\circ} 17^{\prime} 33^{\prime \prime} \mathrm{S}$ de latitude, $45^{\circ} 10^{\prime} 41^{\prime \prime} \mathrm{W}$ de longitude e com altitude de $904 \mathrm{~m}$. O plantio florestal foi implementado com espaçamento de $5 \times 5 \mathrm{~m}$. De cada árvore, foram retiradas duas toras a partir da base cujo comprimento de $0,60 \mathrm{~m}$.

As lâminas de madeira foram obtidas em torno laminador, após o aquecimento das toras à temperatura de 70 ${ }^{\circ} \mathrm{C}$, por período de $24 \mathrm{~h}$ em água, de acordo com recomendações de Iwakiri (2005). Elas foram geradas com espessura nominal de $2 \mathrm{~mm}$ e guilhotinadas nas dimensões de 500 x $500 \mathrm{~mm}$ (largura x comprimento). A massa específica aparente média das lâminas foi de $0,675 \mathrm{~g} \cdot \mathrm{cm}^{-3}$. As lâminas passaram por secagem em estufa com circulação forçada de ar à temperatura de $105 \pm 3{ }^{\circ} \mathrm{C}$, até o teor de umidade médio de $8 \%$. 
Anteriormente à produção dos painéis, classificou-se as lâminas por meio da técnica de excitação por impulso, mediante a utilização do equipamento Sonelastic da empresa ATCP. O equipamento consiste em um suporte ajustável para barras no qual foram posicionadas as lâminas e estimuladas mecanicamente por um pulsador, produzindo uma onda sonora que se propagou pela lâmina e logo captadas por microfone (CARRASCO et al., 2017). Processou-se as informações obtidas por meio do software da ATCP, sendo determinada a constante elástica. As lâminas foram caracterizadas com relação ao modo de vibração flexional. As lâminas com maiores valores de módulo de elasticidade dinâmico (MOEd) foram empregadas nas faces externas, e as de menores MOEd no miolo dos painéis PLP.

\section{Produção dos painéis PLP}

Os painéis foram compostos por sete lâminas, todas elas dispostas na mesma direção da grã. Os adesivos utilizados para colagem dos painéis foram o resorcinol-formaldeído (Cascophen RS 216-M) com adição de 15\% de catalisador (F60-M) (em relação à massa comercial do adesivo) e o poliuretano vegetal (à base de óleo de mamona), bicomponente, em proporção de 1:1,5 do componente A (prépolímero) para o componente B (polyol). O adesivo foi aplicado manualmente com espátula sobre a superfície das lâminas superior e inferior.

O planejamento experimental utilizado para a produção dos painéis PLP de Hevea brasiliensis consistiu no emprego de três diferentes gramaturas para cada tipo de adesivo avaliado, sendo 240,280 e 320 g.m ${ }^{-2}$, resultando em seis tratamentos.

Para a caracterização dos adesivos utilizados após a adição do catalisador, as propriedades de viscosidade, teor de sólidos e potencial hidrogeniônico $(\mathrm{pH})$, dos dois tipos de adesivos foram determinadas e apresentadas na Tabela 1. Para cada propriedade foram realizadas quatro repetições.
A viscosidade foi determinada com uso de um viscosímetro do tipo Copo Ford (com furo de $4 \mathrm{~mm}$ de diâmetro) e os parâmetros estipulados pela norma D1200 (American Society for Testing and Materials- ASTM, 2018a).

A determinação do teor de sólidos foi realizada conforme procedimento descrito por Iwakiri (2005), onde $1 \mathrm{~g}$ do adesivo de cada tratamento foi seco em estufa à temperatura de $105 \pm 3{ }^{\circ} \mathrm{C}$, por três horas. Antes da obtenção da massa do material seco este permaneceu em dessecador por 15 minutos. O percentual do teor de sólidos foi calculado pela razão da massa final em relação a inicial e transformada para percentil. Determinou-se o pH dos adesivos pelo pHmetro, após um período de 4 minutos.

Tabela 1. Propriedades físico-químicos dos adesivos avaliados para a produção dos painéis PLP de Hevea brasiliensis.

Table 1. Physico-chemical properties of the adhesives evaluated for the production of the PLP panels of Hevea brasiliensis.

\begin{tabular}{ccc}
\hline Propriedades & $\begin{array}{c}\text { Resorcinol- } \\
\text { formaldeído }\end{array}$ & $\begin{array}{c}\text { Poliuretano } \\
\text { vegetal }\end{array}$ \\
\hline $\begin{array}{c}\text { Viscosidade } \\
\text { cinemática (cP) }\end{array}$ & 500,26 & 430,63 \\
$\begin{array}{c}\text { Teor de sólidos } \\
\text { (\%) }\end{array}$ & 72,75 & 79,43 \\
pH & 7,17 & 7,00 \\
\hline
\end{tabular}

Realizou-se a prensagem com aplicação de $1 \mathrm{MPa}$ de pressão por $24 \mathrm{~h}$ à temperatura ambiente, os painéis foram acondicionados em câmara climática com temperatura de 20 $\pm 2{ }^{\circ} \mathrm{C}$ e umidade relativa de $65 \pm 5 \%$ por uma semana até a estabilização.

\section{Ensaios dos corpos de prova}

$\mathrm{Na}$ avaliação das propriedades físicas, foram retirados quatro corpos de prova por painel para densidade aparente, umidade e absorção de água, determinados de acordo com a Norma Brasileira Regulamentadora (NBR) 9485 (ASSOCIAÇÃO BRASILEIRA DE NORMAS TÉCNICAS ABNT, 2011). 
Para a determinação da densidade aparente e umidade, foram utilizados corpos de prova nas dimensões 50 x 50 x 18 $\mathrm{mm}$ (comprimento $\mathrm{x}$ largura $\mathrm{x}$ espessura); na absorção de água foram utilizados corpos de prova nas dimensões $75 \times 25$ x $18 \mathrm{~mm}$ (comprimento x largura x espessura).

Em razão das dimensões limitadas dos painéis, em função do tamanho da prensa, adaptações foram feitas para as dimensões dos corpos de prova de compressão paralela com 150 x 50 x $18 \mathrm{~mm}$ (comprimento x largura x espessura). Para a determinação da dureza Janka dos painéis, foram utilizados corpos de prova nas dimensões $75 \times 25 \times 18$ mm (comprimento $\mathrm{x}$ largura $\mathrm{x}$ espessura).

Para os ensaios mecânicos, foram retirados cinco corpos de prova para determinação da rigidez e resistência à compressão paralela às fibras de acordo com a norma D5456 (ASTM, 2018b) e quatro corpos de prova para dureza Janka utilizada a norma D143 (ASTM, 2014) para madeira sólida. Os ensaios mecânicos foram realizados em máquina universal de ensaios dotada de uma célula de carga de $300 \mathrm{kN}$, conectada a um microcomputador para aquisição dos dados.

Já na determinação da delaminação dos painéis PLP, foram esquadrejados quatro corpos de prova, sendo utilizada adaptação descrita abaixo da metodologia referente à norma T110 (American Institute of Timber Construction - AITC, 2007). O ensaio foi realizado em três ciclos, sendo que, em cada ciclo as amostras permaneceram $8 \mathrm{~h}$ imersas em água e, posteriormente $16 \mathrm{~h}$ em estufa com circulação forçada de ar a 60 ㄷ. Após a realização do ensaio, foi verificado o comportamento dos painéis PLP expostos ao ambiente exterior, avaliando a porcentagem de delaminação, que foi obtida nas duas faces de topo dos corpos de prova. O comprimento de cada linha de cola no sentido longitudinal em que ocorreu delaminação foi dividido pelo comprimento total da linha de cola, conforme Equação 1.

$$
\mathrm{Del}=(\text { CompDel } / \text { CompTotal }) * 100 \quad(\text { Equação } 1)
$$

Em que: Del = Delaminação (\%); CompDel = Comprimento em que ocorreu a delaminação $(\mathrm{cm})$; CompTotal $=$ Comprimento total da linha de cola $(\mathrm{cm})$.

\section{Análise estatística}

Os resultados foram submetidos à análise estatística por meio de ANOVA. Rejeitada a hipótese de nulidade, foi aplicada a comparação de médias por meio do teste de Tukey. Realizou-se teste de normalidade por meio do método Shapiro-Wilk. Todos os testes foram efetuados no programa Sisvar 5.6 a $95 \%$ de probabilidade.

\section{Resultados e Discussão}

Observa-se que não houve efeito estatístico entre os tratamentos para umidade, densidade aparente $\mathrm{e}$ delaminação (Tabela 2), sendo a média geral de 10,50 $(0,10)$ $\%$ para umidade, $0,717(0,01)$ g.cm ${ }^{-3}$ para densidade aparente e $4,65(1,85) \%$ para delaminação.

Segundo Fiorelli; Dias (2005), é de extrema importância a realização de ensaios para avaliar a qualidade da ligação adesiva quando a mesma é submetida a variações de umidade, pressão e temperatura, visando garantir a integridade do elemento estrutural durante a vida útil da construção.

Tabela 2. Valores médios para umidade, densidade aparente, delaminação e absorção de água dos painéis PLP de Hevea brasiliensis produzidos com três diferentes teores de adesivos.

Table 2. Mean values for moisture, apparent density, delamination and water absorption of PLP panels of Hevea brasiliensis produced with three different adhesive contents.

\begin{tabular}{|c|c|c|c|c|}
\hline Tratamento & Umidade (\%) & Densidade aparente $\left(\mathrm{g} \cdot \mathrm{cm}^{-3}\right)$ & Delaminação (\%) & Absorção de água (\%) \\
\hline$R F \_240$ & $10,48(0,18) A^{*}$ & $0,698(0,19) \mathrm{A}$ & $3,38(1,77) \mathrm{A}$ & $37,57(5,45) \mathrm{B}$ \\
\hline$R F \_280$ & $10,57(0,12) \mathrm{A}$ & $0,704(0,14) \mathrm{A}$ & $7,25(2,05) \mathrm{A}$ & $39,60(2,78) \mathrm{B}$ \\
\hline$R F \_320$ & $10,47(0,20) \mathrm{A}$ & $0,725(0,25) \mathrm{A}$ & $6,82(0,74) \mathrm{A}$ & $35,69(2,52) \mathrm{B}$ \\
\hline$P V \_240$ & $10,48(0,41) \mathrm{A}$ & $0,719(0,16) \mathrm{A}$ & $3,69(1,69) \mathrm{A}$ & $36,12(4,23)$ B \\
\hline
\end{tabular}




$\begin{array}{lllll}P V \_280 & 10,34(0,20) \mathrm{A} & 0,714(0,22) \mathrm{A} & 3,55(3,25) \mathrm{A} & 28,76(6,43) \mathrm{A} \\ \boldsymbol{P V} \text { _320 } & 10,65(0,32) \mathrm{A} & 0,744(0,14) \mathrm{A} & 3,21(2,48) \mathrm{A} & 27,96(2,36) \mathrm{A}\end{array}$

${ }^{*}$ Médias seguidas de mesma letra em mesma coluna são estatisticamente iguais, pelo teste de Tukey a 95\% de probabilidade. Desvio padrão entre parênteses. RF_240, RF_280 e RF_320: referem-se à painéis produzidos com adesivo resorcinolformaldeído nas gramaturas 240 g.m $\mathrm{m}^{-2}, 280$ g.m $\mathrm{m}^{-2}$ e 320 g.m $\mathrm{m}^{-2}$, respectivamente. PV_240, PV_280 e PV_320: referem-se à painéis produzidos com adesivo poliuretano vegetal nas gramaturas 240 g.m $\mathrm{m}^{-2}, 280$ g.m $\mathrm{m}^{-2}$ e 320 g.m $\mathrm{m}^{-2}$, respectivamente.

Kamala et al. (1999) estudando as propriedades físicas e mecânicas de painéis PLP produzidos com adesivo fenolformaldeído e madeira de Hevea brasiliensis encontraram valores inferiores à 5\% para resistência à delaminação. Lahr et al. (2007) avaliaram a porcentagem de delaminação em painéis PLP produzidos com madeira de um híbrido entre as espécies Eucalyptus grandis Hill ex Maiden e Eucalyptus urophylla S.T. Blake unidos com adesivo fenol-formaldeído, onde obtiveram resistência à delaminação de 5,33\%. Salientase que o adesivo utilizado por ambos pesquisadores possui função à prova d'água, mesmo comportamento apresentado pelos adesivos resorcinol-formaldeído e poliuretano vegetal. Os resultados encontrados neste trabalho para resistência à delaminação encontram-se inferiores ao máximo de 10\% estipulado pela norma europeia EN 386 (European Standard, 2001).

$\mathrm{Na}$ Figura 1 apresenta-se fendas da delaminação característica em ambas as faces dos painéis PLP colados com RF para a gramatura 240 g.m $\mathrm{m}^{-2}$.

Para absorção de água dos painéis PLP, nota-se que houve diferença estatística, em que os tratamentos PV_280 e PV_320 apresentaram os menores valores para essa propriedade física. Esse comportamento pode ser explicado pela viscosidade dos adesivos, como o adesivo PV apresenta viscosidade menor e é mais fluido que o RF, o mesmo espalhou melhor pela superfície das lâminas, preenchendo os poros e resultando em processo adesivo satisfatório. $\mathrm{O}$ tratamento PV_240 apresentou valores médios estatisticamente iguais aos tratamentos produzidos com RF, esse fato pode ser atribuído a uma formação de linha de cola faminta, devido à menor gramatura aplicada de adesivo PV. Com exceção dos supracitados, os demais apresentaram teores de absorção de água superiores aos determinados por Guimarães Júnior et al. (2015), que estudou 5 clones de Eucalyptus urophylla para produção de painéis PLP com adesivo resorcinol-formaldeído na gramatura de 320 g.m ${ }^{-2}$, obtiveram valor médio de absorção de água de $26,88 \%$.

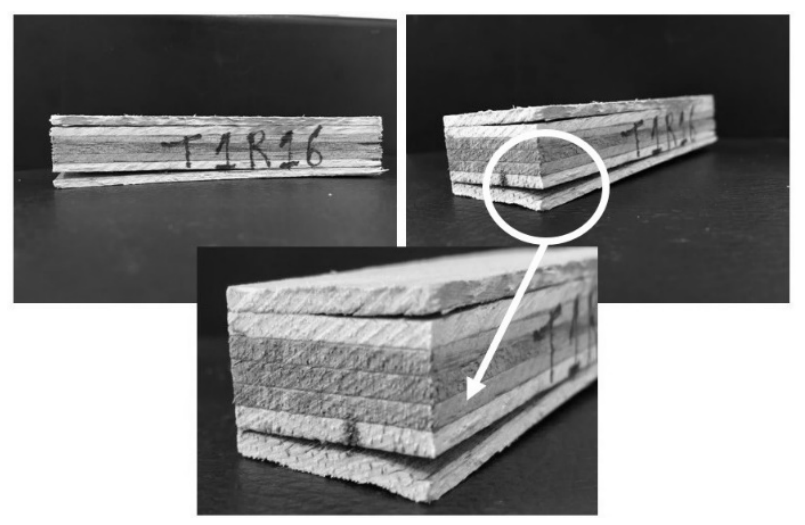

Figura1. Fendas características após ensaio da delaminação dos painéis PLP de Hevea brasiliensis colados com RF.

Figure 1. Characteristic cracks after delamination test of LVL panels of Hevea brasiliensis glued with RF.

Os maiores valores de absorção de água encontrados neste trabalho em relação aos obtidos por Guimarães Júnior et al. (2015) podem ser explicados devido aos maiores valores da densidade aparente encontrado pelos autores, cujos valores variaram entre 0,720 e 0,830 g.cm ${ }^{-3}$. A densidade geral média das chapas produzidas pelos autores ficou superior, em aproximadamente, 8,38\% em comparação aos painéis PLP da madeira de Hevea brasiliensis. Maiores teores de absorção de água também se devem a maior taxa de holocelulose presente na madeira de Hevea brasiliensis, uma vez que esta espécie possui cerca de 75\% de holocelulose (RAIA et al., 2018), enquanto a madeira de Eucalyptus urophylla possui cerca de 62\% (TRUGILHO et al., 2015), como essa estrutura da parede celular possui caráter hidrofílico, promove maior absorção de água ao painel PLP. 
A absorção de água observada para os painéis de Hevea brasiliensis deste trabalho foi inferior ao determinado no trabalho de Mendoza et al. (2017), que desenvolveu painéis PLP de Trattinnickia burserifolia com densidade aparente média de $0,525 \mathrm{~g} . \mathrm{cm}^{-3}$, as lâminas foram coladas com resina sintética à base de fenol-formaldeído na gramatura de 280 g.m-2 , cujo valor médio para absorção de água foi de $68,23 \%$. A Trattinnickia burserifolia é uma madeira que apresenta alta porosidade, o que pode ter favorecido a entrada de água na superfície e o aumento de massa, favoreceu maior absorção de água percentual. São desejados menores valores para essa propriedade física, portanto, esse resultado reforça o potencial de uso de madeira de Hevea brasiliensis para a produção de painéis PLP.

Não houve diferença estatística para a resistência à compressão paralela dos painéis PLP (Tabela 3), apresentou valor médio geral de 49,50 (1,97) $\mathrm{MPa}$.

Tabela 3. Valores médios de resistência e rigidez no ensaio de compressão paralela às fibras e dureza Janka.

Table 3. Mean values of stiffness and strength in the compression parallel to the fibers and Janka hardness tests.

\begin{tabular}{|c|c|c|c|}
\hline \multirow{2}{*}{ Tratamento } & \multicolumn{2}{|c|}{ Compressão Paralela } & \multirow{2}{*}{$\begin{array}{c}\text { Dureza Janka } \\
\mathrm{MPa}\end{array}$} \\
\hline & $\mathrm{f}_{\mathrm{c} 0}(\mathrm{MPa})$ & $\mathrm{E}_{\mathrm{c} 0}(\mathrm{MPa})$ & \\
\hline$R F \_240$ & $\begin{array}{c}52,28 \\
(4,57) A^{*}\end{array}$ & $\begin{array}{c}3406,98 \\
(321,75) \mathrm{A}\end{array}$ & $33,30(0,34) \mathrm{A}$ \\
\hline$R F \_280$ & $\begin{array}{c}48,26 \\
(6,34) \mathrm{A}\end{array}$ & $\begin{array}{c}4237,87 \\
(475,19) \mathrm{A}\end{array}$ & $36,32(0,13) \mathrm{A}$ \\
\hline$R F \_320$ & $\begin{array}{c}46,76 \\
(3,64) \mathrm{A}\end{array}$ & $\begin{array}{c}6304,60 \\
(270,15) \mathrm{B}\end{array}$ & $34,69(0,59) \mathrm{A}$ \\
\hline$P V \_240$ & $\begin{array}{c}50,71 \\
(2,78) \mathrm{A}\end{array}$ & $\begin{array}{c}4528,95 \\
(365,02) \mathrm{A}\end{array}$ & $36,34(0,33) \mathrm{A}$ \\
\hline PV_280 & $\begin{array}{c}50,27 \\
(4,25) \mathrm{A}\end{array}$ & $\begin{array}{c}3822,42 \\
(247,37) \mathrm{A}\end{array}$ & $35,10(0,46) \mathrm{A}$ \\
\hline$P V \_320$ & $\begin{array}{c}48,73 \\
(4,94) \mathrm{A}\end{array}$ & $\begin{array}{c}5416,77 \\
(389,24) \mathrm{B}\end{array}$ & $37,79(0,61) \mathrm{A}$ \\
\hline
\end{tabular}

Em que: $\mathrm{f}_{\mathrm{c} 0}=$ resistência à compressão paralela às fibras; $\mathrm{E}_{\mathrm{c} 0}=$ rigidez à compressão paralela às fibras. ${ }^{\star}$ Médias seguidas de mesma letra em mesma coluna são estatisticamente iguais, pelo teste de Tukey a $95 \%$ de probabilidade. Desvio padrão entre parênteses. RF_240, RF_280 e RF_320: referem-se à painéis produzidos com adesivo resorcinol-formaldeído nas gramaturas 240 g.m $\mathrm{m}^{-2}, 280$ g.m $\mathrm{m}^{-2}$ e 320 g.m $\mathrm{m}^{-2}$, respectivamente. PV_240, PV_280 e PV_320: referem-se à painéis produzidos com adesivo poliuretano vegetal nas gramaturas 240 g.m ${ }^{-2}$, 280 g. $\mathrm{m}^{-2}$ e 320 g. $\mathrm{m}^{-2}$, respectivamente.

Resultados semelhantes foram observados por Kamala et al. (1999) estudando as propriedades físicas e mecânicas de painéis PLP de Hevea brasiliensis produzidos com adesivo fenol-formaldeído, determinaram valor médio de 47,36 MPa. Já para rigidez à compressão paralela dos painéis PLP, os tratamentos RF_320 e PV_320 apresentaram os maiores valores médios. Tais resultados podem ser explicados pela maior gramatura dos painéis em questão, onde os tratamentos foram colados com gramatura de 320 g.m ${ }^{-2}$, resultando em uma linha de cola mais rígida, promovendo uma melhor densificação dos painéis. Comparando os valores obtidos neste trabalho aos encontrados por Lara Palma; Ballarin (2011), observa-se que a rigidez à compressão paralela dos painéis PLP de Eucalyptus grandis produzidos com adesivo fenol-formaldeído na gramatura de 350 g.m ${ }^{-2}$ dos autores ficaram muito superiores, com valor médio de $16856 \mathrm{MPa}$.

Para dureza Janka, não houve diferença estatística entre os tratamentos avaliados, que apresentaram média geral de 35,50 (1,56) MPa. Os resultados se mostraram satisfatórios, uma vez que a dureza Janka observada no presente trabalho se apresentou compatível com a da madeira sólida, podendo ser aplicada em locais que a resistência superficial é requerida, desde que os painéis não fiquem expostos ao ambiente exterior. 
Tais resultados obtidos nesse trabalho para dureza Janka encontram-se similares aos obtidos por Buligon et al. (2018), com valor médio de 46,67 MPa para painéis LVL produzidos com resina fenol-formaldeído na gramatura de 190 g.m $\mathrm{m}^{-2} \mathrm{em}$ linha simples. Já Delucis et al. (2018) obtiveram valores superiores para as propriedades de painéis PLP produzidos com as madeiras de Eucalyptus saligna e Pinus taeda e adesivo fenol-formaldeído na gramatura de 200 g.m², obtiveram valor geral médio de $65 \mathrm{MPa}$. Embora a dureza Janka seja um teste mecânico realizado na superfície da madeira, os resultados obtidos descrevem mecanismos (interações da linha de cola entre as lâminas) que ocorrem nas camadas internas dos painéis (DELUCIS et al., 2018). Ainda segundo os autores, esse fato pode ser atribuído à propagação de tensões através das interfaces das lâminas e formação de fissuras, já que a carga de tração realizada durante o teste mecânico também conduz carga de compressão, logo, a rigidez total do painel tem um papel importante.

\section{Conclusões}

Com base nos resultados obtidos, as seguintes conclusões podem ser apresentadas:

Em relação aos ensaios físicos realizados nos painéis PLP, não houve diferenças estatísticas para umidade e densidade aparente para os tratamentos estudados. Já para absorção de água, o adesivo poliuretano vegetal apresentou os menores teores para esta propriedade.

Para as propriedades mecânicas, os painéis PLP apresentaram resultados satisfatórios para os ensaios realizados, onde mostrou-se diferença apenas para a rigidez no ensaio de compressão paralela às fibras.

A resistência à delaminação dos painéis atende aos requisitos da Norma Europeia EN 386.

Os resultados indicam a viabilidade de uso de lâminas de Hevea brasiliensis para a fabricação de painéis PLP, podendo ser empregados em pisos, flanges de vigas "I", degraus de escada e pontes.

\section{Agradecimentos}

Os autores agradecem à empresa Imperveg pela doação do Poliuretano Vegetal. À Fundação de Amparo à Pesquisa do Estado de Minas Gerais - FAPEMIG, Coordenação de Aperfeiçoamento de Pessoa de Nível Superior - CAPES, Conselho Nacional de Desenvolvimento Científico e Tecnológico - CNPq e ao Sr. Vádi por gentilmente permitir a realização desta pesquisa em seu plantio florestal de seringueira.

\section{Referências}

AMERICAN INSTITUTE OF TIMBER CONSTRUCTION (AITC). T110: Test methods for structural glued laminated timber. Colorado, 2007.

AMERICAN SOCIETY FOR TESTING AND MATERIALS (ASTM). D143: Methods of testing of small clear specimens. West Conshohocken, 2014.

AMERICAN SOCIETY FOR TESTING AND MATERIALS (ASTM). D1200: Standard test method for viscosity by Ford viscosity cup. West Conshohocken, 2018a.

AMERICAN SOCIETY FOR TESTING AND MATERIALS (ASTM). D5456: Standard specification for evaluation of structural composite lumber products. West Conshohocken, 2018 b.

ASSOCIAÇÃO BRASILEIRA DE NORMAS TÉCNICAS (ABNT). NBR 9485: Compensado - Determinação da massa específica aparente. Rio de Janeiro, 2011.

BERGER, C.; PERTUZATTI, A.; HASELEIN, C. R. Influência da posição de lâminas de duas espécies exóticas nas propriedades mecânicas de painéis LVL. Ciência da Madeira, v. 9, n. 1, p. 19-29, 2018.

BUligON, E. A.; HASElEIN, C. R.; GATTO, D. A.; SANTINI, E. J.; RAUBER, R.; BERGER, C. Propriedades físico-mecânicas de painéis de lâminas paralelas reforçados com PRFV. Ciência Florestal, v. 25, n. 3, p. 731-741, 2015.

BUligON, E. A.; HASELEIN, C. R.; TEIXEIRA, D. E.; WIMMER, P.; MANAVELLA, C. Avaliação da Dureza Janka de Painéis de Lâminas Paralelas (LVL) Reforçados com Fibra de Vidro. In: XVI Encontro Brasileiro em Madeiras e em Estruturas de Madeira e III Congresso Latinoamericano de Estruturas de Madeira, 2018, São Carlos, 11p. 
CARRASCO, E. V. M.; VARGAS, C. B.; SOUZA, M. F.; MANTILLA, J. N. R. Avaliação das características mecânicas da madeira por meio de excitação por impulso. Revista Matéria, v. 22, supl. 1, 2017.

DELUCIS, R.; HERRERA, R.; MELO, R.; MULLER, M.; LABIDI, J.; GATTO, D. Hybrid LVL panels made of eucalypt and pine woods decayed by White-rot fungus. Drewno, v. 61, n. 201, 2018.

EUROPEAN STANDARD. EN 386: Glued Laminated Timber: Performance requirements and minimum production requirements. 2001.

FIORELLI, J.; DIAS, A. A. Avaliação do comportamento da madeira na compressão paralela às fibras e sua influência na resistência de vigas de MLC reforçadas com PRF. Revista Madeira, v. 1, p. 1-15, 2005.

GUIMARÃES JÚNIOR, J. B.; PROTÁSIO, T. P.; MENDES, R. F.; MENDES, L. M.; GUIMARÃES, B. M. R.; SIQUEIRA, H. F. Quality of LVL panels produced with wood from Eucalyptus urophylla clones. Pesquisa Florestal Brasileira,v. 35, n. 83, p. 307-313, 2015.

IWAKIRI, S. Painéis de madeira reconstituída. Curitiba: FUPEF, 2005. 247 p.

IWAKIRI, S.; MATOS, J. L. M.; PRATA, J. G.; TORQUATO, L. P.; BRONOSKI, M.; NISHIDATE, M. M. Produção de painéis laminados unidirecional - LVL com madeiras de Eucalyptus grandis Hill ex Maiden e Eucalyptus dunnii Maiden. Floresta e Ambiente, v. 15, n. 2, p. 01-07, 2008.

IWAKIRI, S.; TRIANOSKI, R.; PRATA, J. G.; MULLER, B. V.; CARVALHO, D. E.; FREITAS JÚNIOR, J. A.; ROSA, T. S. Evaluation of potential for using six species of the genus Eucalyptus in the production of laminated veneer lumber LVL. Scientia Forestalis, v. 44, n. 110, p. 351-359, 2016.

KAMALA, B. S.; KUMAR, P.; RAO, R. V.; SHARMA, S. N. Performance test of Laminated Veneer Lumber (LVL) from rubber wood for different physical and mechanical properties. Holz als Roh-und Werkstoff, v. 57, p. 114-116, 1999.

LAHR, F. A. R.; CARVALHO, A. M.; MACHADO, J. M. S. Painéis LVL de Eucalipto estimativa da porcentagem de delaminação. Revista Madeira, n. 21, 2007.

LARA PALMA, H. A.; BALLARIN, A. W. Propriedades físicas e mecânicas de painéis LVL de Eucalyptus grandis. Ciência Florestal, v. 21, n. 3, p. 559-566, 2011.
MENDOZA, Z. M. S. H.; BORGES, P. H. M.; SANTOS, E. A.; PENNA, J. E.; ELIAS, M. P. S.; MORAIS, P. H. M. Estudo comparativo das propriedades físicas e mecânicas de painéis compensados e laminated veneer lumber (LVL). Nativa, v. 5, p. 588-593, 2017.

MÜlLER, M. T.; HASELEIN, C. R.; MELO, R. R. M.; STANGERLIN, D. M. Influência de diferentes combinações de lâminas de Eucalyptus saligna e Pinus taeda em painéis LVL. Ciência Florestal, v. 25, n. 1, p. 153-164, 2015.

RAIA, R. Z.; IWAKIRI, S.; TRIANOSKI, R.; ANDRADE, A. S.; BONFATTI JUNIOR, E. A. Influência da extração de látex nas propriedades físicas e químicas da madeira de Hevea brasiliensis. Ciência da Madeira, v. 9, n. 3, p. 152-159, 2018.

TRUGILHO, P. F.; GOULART, S. L.; ASSIS, C. O.; COUTO, F. B. S.; ALVES, I. C. N.; PROTÁSIO, T. P.; NAPOLI, A. Características de crescimento, composição química, física e estimativa de massa seca de madeira em clones e espécies de Eucalyptus jovens. Ciência Rural, v. 45, n. 4, p. 661-666, 2015. 